\title{
Software-Defined-Radio-Assisted Adaptive Broadband Frequency Hopping Multicarrier DS-CDMA
}

\author{
Lie-Liang Yang and Lajos Hanzo, University of Southampton
}

\begin{abstract}
Against the backdrop of the explosive expansion of the Internet and the continued dramatic increase in demand for high-speed multimedia wireless services, there is an urgent demand for flexible, bandwidth-efficient transceivers. Multistandard operation is also an important requirement for the future generations of wireless systems. This article presents a versatile broadband multiple access scheme, combining frequency hopping with multicarrier DS-CDMA (FH/MC DS-CDMA). In the short term, the proposed FH/MC DS-CDMA scheme could be employed in the existing second- and third-generation CDMA system bands. Hence, it has the potential of supporting compatibility with these existing systems. This article also shows that FH/MC DS-CDMA exhibits a high grade of flexibility in the context of system design and parameter reconfiguration. Therefore, in the long term, the proposed FH/MC DS-CDMA scheme is capable of meeting the requirements of future broadband mobile wireless systems, with the aid of introducing more advanced techniques facilitated by the employment of software-defined radios (SDR) and with the advent of efficient adaptive baseband algorithms.
\end{abstract}

\section{INTRODUCTION}

There are a range of activities in various parts of the globe concerning the standardization, research, and development of the thirdgeneration $(3 \mathrm{G})$ mobile systems termed IMT-2000 by the International Telecommunication Union (ITU) [1, 2]. The most recent version of the IMT-2000 standard in fact constitutes a range of five independent standards. These are the UMTS Terrestrial Radio Access (UTRA) frequency-division duplex (FDD) wideband code-division multiple access (W-CDMA) mode [3], UTRA time-division duplex (TDD) CDMA mode [3], Pan-American multicarrier CDMA configuration mode known as cdma2000 [3],
Pan-American time-division multiple access (TDMA) mode known as UWT-136, and Digital Enhanced Cordless Telecommunications (DECT) [3] mode. It would be desirable to achieve future systems fitting into a generic future-proof framework, such as the proposed flexible system, while additionally offering more flexible and powerful features, without having to define new standards. The framework proposed in this contribution is capable of satisfying this requirement.

In the first part of this treatise a broadband multiple access candidate scheme meeting the above requirements is presented, which is constituted by frequency-hopping (FH)-based multicarrier direct sequence (DS) CDMA (FH/MC DS-CDMA) [4, 5]. Recent investigations demonstrated that channel-quality controlled rate adaptation is an efficient strategy for attaining the highest possible spectral efficiency in terms of bits per second per Hertz [6, 7], while maintaining a certain target integrity. Hence, in the second part of the article we consider adaptive rate transmission (ART) schemes associated with supporting both time-variant-rate and multirate services. These ART techniques are discussed in the context of the proposed FH/MC DS-CDMA system, arguing that software-difined radios (SDRs) constitute a convenient framework for their implementation. Therefore, in the final part of this contribution the concept of SDRassisted broadband FH/MC DS-CDMA is presented, and the range of reconfigurable parameters are described with the aim of outlining a set of promising research topics. Let us now commence our detailed discourse concerning the proposed $\mathrm{FH} / \mathrm{MC}$ DS-CDMA system.

\section{FH/MC DS-CDMA}

The transmitter schematic of the proposed FH/MC DS-CDMA arrangement is depicted in Fig. 1. Each subcarrier of a user is assigned a pseudo-noise (PN) spreading sequence. These PN sequences can be simultaneously assigned to 


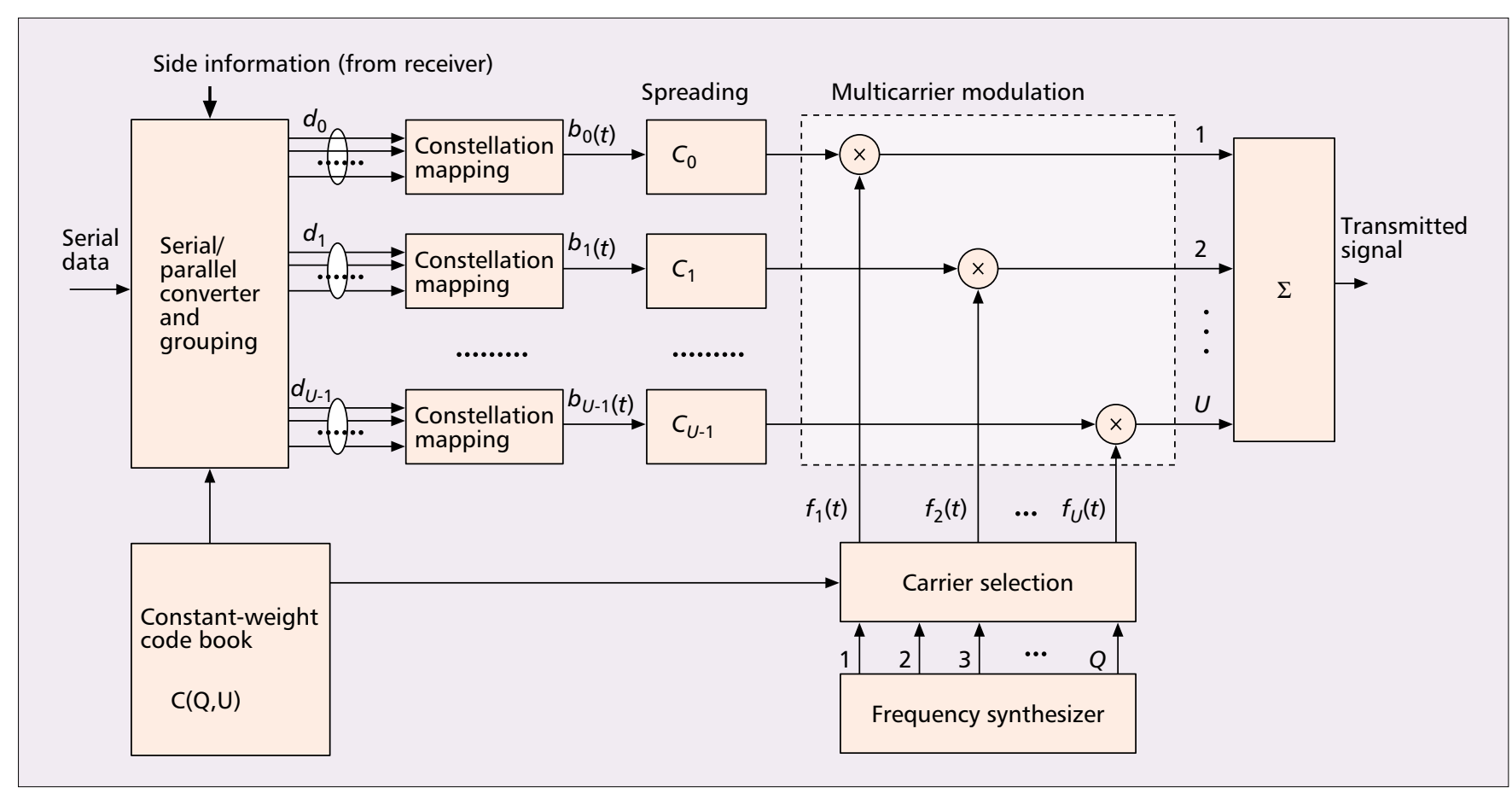

Figure 1. Transmitter diagram of the FH/MC DS-CDMA system using adaptive transmission.

a number of users, provided that no more than one user activates the same PN sequence on a given subcarrier. These PN sequences produce narrowband DS-CDMA signals. In Fig. $1, C(Q$, $U)$ represents a constant-weight code having $U$ number of 1 s and $(Q-U)$ number of 0 s. Hence, the weight of $\mathrm{C}(Q, U)$ is $U$. This code is read from a so-called constant-weight code book, which represents the frequency-hopping patterns. The constant-weight code $C(Q, U)$ plays two different roles. Its first role is that its weight - namely $U$ - determines the number of subcarriers invoked, while its second function is that the positions of the $U$ number of binary $1 \mathrm{~s}$ determines the selection of a set of $U$ number of subcarrier frequencies from the $Q$ outputs of the frequency synthesizer. Furthermore, in the transmitter, side information reflecting the channel's instantaneous quality might be employed, in order to control its transmission and coding mode so that the required target throughput and transmission integrity requirements are met.

As shown in Fig. 1, the original bitstream having a bit duration of $T_{b}$ is first serial-to-parallel (S-P) converted. Then these parallel bit streams are grouped and mapped to the potentially time-variant modulation constellations of the $U$ active subcarriers. Let us assume that the number of bits transmitted by an FH/MC DSCDMA symbol is $M$, and let us denote the symbol duration of the FH/MC DS-CDMA signal by $T_{s}$. Then, if the system is designed for achieving a high processing gain and mitigating the intersymbol interference (ISI) in a constant-rate transmission scheme, the symbol duration can be extended to a multiple of the bit duration (i.e., $T_{s}=M T_{b}$ ). In contrast, if the design aims to support multiple transmission rates or channel-quality matched variable information rates, a constant symbol duration of $T_{0}$ may be employed. Both multirate and variable-rate transmissions can be implemented by employing a different number of subcarriers associated with different modulation constellations as well as different spreading gains. As seen in Fig. 1, after the constellation mapping stage, each branch is DS spread using the assigned PN sequence, and then this spread signal is carrier modulated using one of the active subcarrier frequencies derived from the constant-weight code $C(Q, U)$. Finally, all $U$ active branch signals are multiplexed in order to form the transmitted signal.

In the FH/MC DS-CDMA receiver of Fig. 2 the received signal associated with each active subcarrier is detected using, for example, a RAKE combiner. Alternatively, multiuser detection (MUD) can be invoked in order to approach the single-user bound. In contrast to the transmitter side, where only $U$ out of $Q$ subcarriers are transmitted by a user, at the receiver different detector structures might be implemented based on the availability [4] or lack [5] of the $\mathrm{FH}$ pattern information. During the FH pattern acquisition stage, which usually happens at the beginning of transmission or during handover, tentatively all $Q$ subcarriers can be demodulated. The transmitted information can be detected and the FH patterns acquired simultaneously by using blind joint detection algorithms exploiting the characteristics of the constant-weight codes [5]. If, however, the receiver has explicit knowledge of the $\mathrm{FH}$ patterns, only $U$ subcarriers have to be demodulated. However, if fast Fourier transform (FFT) techniques are employed for demodulation, as is often the case in MC CDMA or OFDM systems, all $Q$ subcarriers might be demodulated, where the inactive subcarriers only output noise. In the decision unit of Fig. 2, these noise-output-only branches can be eliminated by exploiting knowledge of the FH patterns. Hence, 


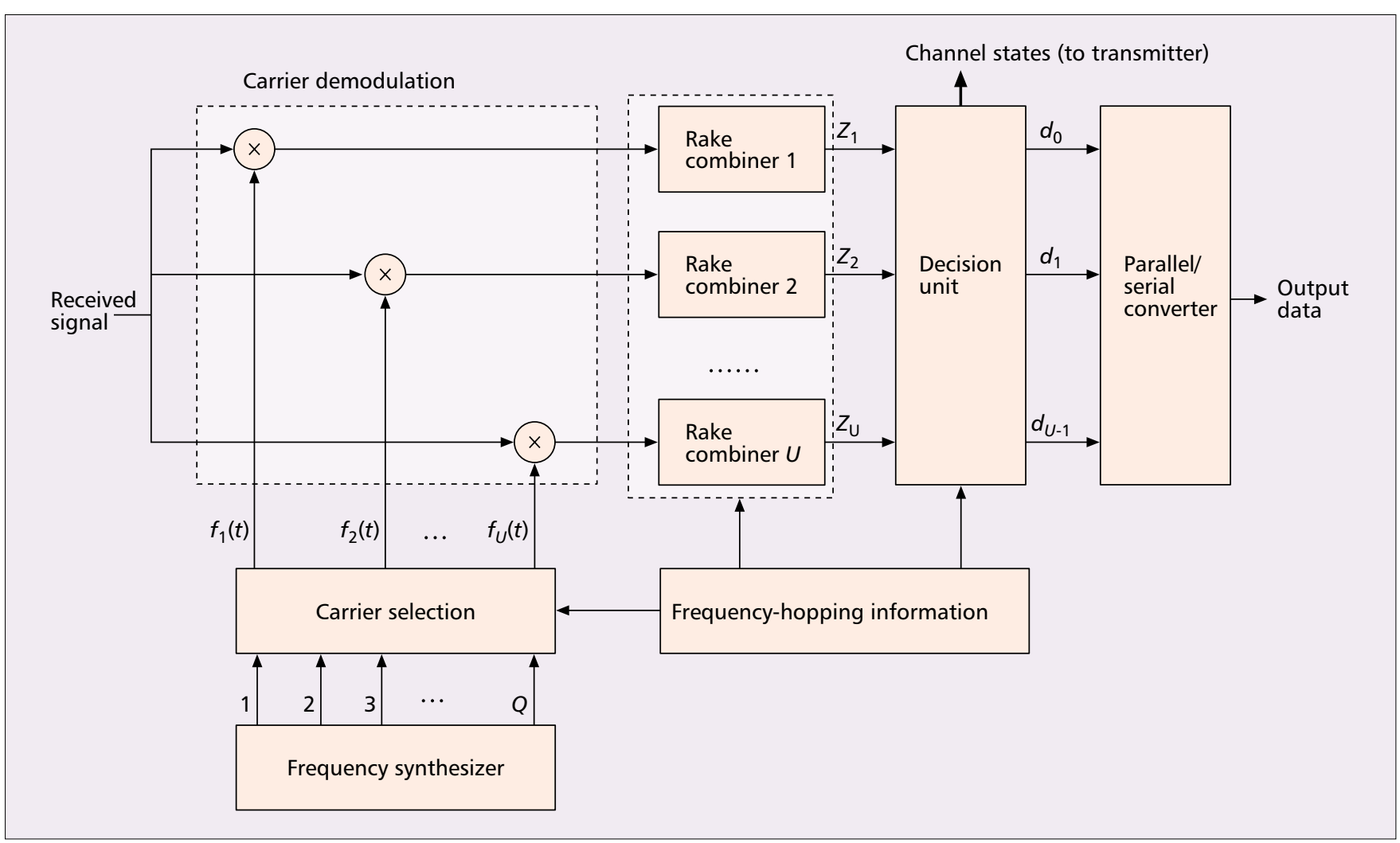

Figure 2. A receiver block diagram of the FH/MC DS-CDMA system using a conventional RAKE receiver.

the decision unit only outputs the information transmitted by the active subcarriers. Finally, the decision unit's output information is parallel-toserial converted to form the output data.

At the receiver, the channel states associated with all the subchannels might be estimated or predicted using pilot signals $[6,7]$. This channel state information can be utilized for coherent demodulation. It can also be fed back to the transmitter as highly protected side-information, in order to invoke a range of adaptive transmission schemes including power control and adaptive-rate transmission.

\section{CHARACTERISTICS OF THE FH/MC DS-CDMA SYSTEMS}

In the proposed FH/MC DS-CDMA system the entire bandwidth of future broadband systems can be divided into a number of subbands, and each subband can be assigned a subcarrier. According to the prevalent service requirements, the set of legitimate subcarriers can be distributed in line with the users' instantaneous information rate requirements. If this $\mathrm{FH} / \mathrm{MC}$ DS-CDMA system were rolled out over the existing $2 \mathrm{G}$ and $3 \mathrm{G}$ CDMA bands and the subbands in the $\mathrm{FH} / \mathrm{MC}$ DS-CDMA scheme were configured based on the requirements of the $2 \mathrm{G}$ and $3 \mathrm{G}$ CDMA systems, in this respect $\mathrm{FH} / \mathrm{MC}$ DS-CDMA would exhibit full compatibility with the existing $2 \mathrm{G}$ and $3 \mathrm{G}$ CDMA systems. In FH/MC DS-CDMA specific FH techniques can be employed for each user, in order to evenly occupy the whole system bandwidth available and efficiently utilize the available frequency resources. Therefore, from a networking performance respective, the average transmission delay and blocking probability can be reduced.

\section{A Broadband Wireless Mobile System}

To elaborate a little further, our advocated FH/MC DS-CDMA system is a broadband wireless mobile system constituted by multiple narrowband DS-CDMA subsystems. Again, FH techniques are employed for each user, in order to evenly occupy the whole system bandwidth and efficiently utilize the available frequency resources. The constant-weight code-based FH patterns used in the schematic of Fig. 1 are invoked in order to control the number of subcarriers invoked, which is kept constant during the $\mathrm{FH}$ procedure. In contrast to broadband singlecarrier DS-CDMA (SC DS-CDMA) systems [2], which inevitably result in extremely high-chip-rate spreading sequences and high complexity, the proposed FH/MC DS-CDMA system does not have to use high-chip-rate DS spreading sequences, since each subcarrier conveys a narrowband DS-CDMA signal. In contrast to broadband OFDM systems, which have to use a high number of subcarriers and usually result in a high peak-to-average power fluctuation, due to the associated DS spreading the number of subcarriers of the advocated broadband wireless $\mathrm{FH} / \mathrm{MC}$ DS-CDMA system may be significantly lower. This potentially mitigates the crest-factor problem. Additionally, with the aid of $\mathrm{FH}$, the peakto-average power fluctuation of the $\mathrm{FH} / \mathrm{MC}$ DS-CDMA system might be further decreased. In other words, the FH/MC DS-CDMA system is capable of combining the best features of SC DSCDMA and orthogonal frequency-division multi- 
plex (OFDM) systems, while avoiding many of their individual shortcomings. Finally, in comparison to the FH/MC DS-CDMA system, both broadband SC DS-CDMA systems and broadband OFDM systems are less amenable to interworking with the existing $2 \mathrm{G}$ - and $3 \mathrm{G}$ wireless communication systems.

\section{COMPATIBILITY}

The broadband FH/MC DS-CDMA system can be rolled out over the bands of $2 \mathrm{G}$ and $3 \mathrm{G}$ mobile wireless systems and/or in the band licensed for future broadband wireless communication systems. In FH/MC DS-CDMA systems the subbands associated with different subcarriers are not required to be of equal bandwidth. Hence existing 2G and 3G CDMA systems can be supported using one or more subcarriers. For example, Fig. 3 shows the spectrum of an $\mathrm{FH}$ orthogonal MC DS-CDMA signal using a subchannel bandwidth of $1.25 \mathrm{MHz}$, which constitutes the bandwidth of a DS-CDMA signal in the IS-95 standard [1]. In Fig. 3 we also show that seven subchannels, each with a bandwidth of $1.25 \mathrm{MHz}$, can be replaced by one subchannel with a bandwidth of $5 \mathrm{MHz}(=8 \times 1.25 / 2$ $\mathrm{MHz}$ ). Hence, the narrowband IS-95 CDMA system can be supported by a single subcarrier, while the UMTS and IMT-2000 W-CDMA systems might be supported using seven subchannels' bandwidth amalgamated into one W-CDMA channel. Moreover, with the aid of SDRs, FH/MC DS-CDMA is also capable of embracing other existing standards, such as the TDMA-based Global System for Mobile Communications (GSM) [3].

\section{FH StRATEgY}

In FH/MC DS-CDMA systems both slow and fast FH techniques can be invoked, depending on the system's design and the state of the art. In slow FH several symbols are transmitted after each FH, while in fast $\mathrm{FH}$ several frequency hops take place in a symbol duration; each symbol is transmitted using several subcarriers, and hence diversity can be achieved by combining these subcarrier signals. Moreover, from a networking point of view, random, uniform, and adaptive FH schemes can be utilized [4] in order to maximize the efficiency of the network. In the context of random $\mathrm{FH}$, the subcarriers associated with each transmission of a user are determined by the set of preassigned FH patterns constituting a group of constant-weight codewords. The active subcarriers are switched from one group of frequencies to another without the knowledge of the FH patterns of the other users. By contrast, for the FH/MC DSCDMA system using uniform $\mathrm{FH}$, the $\mathrm{FH}$ patterns of all users are determined jointly under the control of the base station (BS) so that each subcarrier is activated by a similar number of users. It can be shown that for the downlink uniform $\mathrm{FH}$ can readily be implemented, since the BS has knowledge of the FH patterns of all users. However, for implementing uplink transmissions, the FH patterns to be used must be signaled by the BS to each mobile station (MS) in order to be able to implement uniform FH. Finally, if the near-instantaneous channel quali-

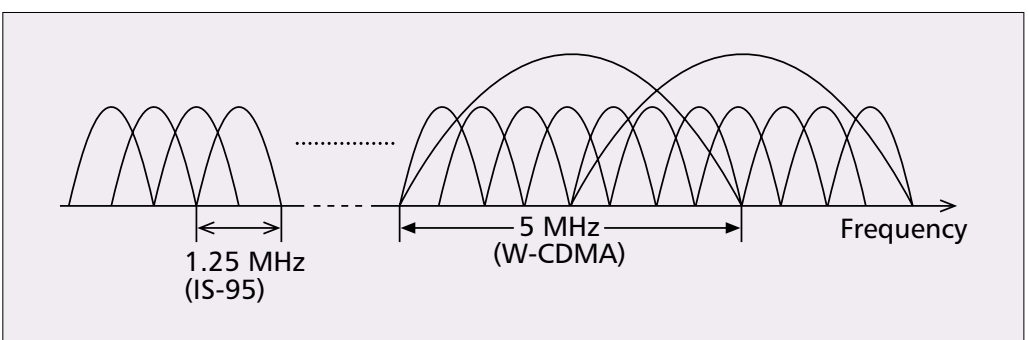

Figure 3. Spectrum of a FH/MC DS-CDMA signal using a subchannel bandwidth of $1.25 \mathrm{MHz}$ and/or $5 \mathrm{MHz}$.

ty information is available at the transmitter, advanced adaptive $\mathrm{FH}$ can be invoked, where information is only transmitted over a group of subchannels exhibiting a satisfactory signal-tointerference ratio (SIR).

\section{IMPLEMENTATION OF MC MODULATION}

The MC modulation and MC demodulation blocks in Fig. 2 can be implemented using FFT techniques, provided that each of the subchannels occupies the same bandwidth. Since not all of the subcarriers are activated at each transmission in the proposed FH/MC DS-CDMA system, the deactivated subcarriers can be set to zero in the FFT or inverse FFT (IFFT) algorithm. However, if an unequal bandwidth is associated with the subchannels, MC modulation/demodulation can only be implemented using less efficient conventional, rather than FFT-based, carrier modulation/demodulation schemes.

\section{New User Access and TRAnsmission}

A new user can access the network through dedicated access channels. When a new user who successfully accessed the network attempts to commence his/her transmission, a range of different strategies might be offered by the FH/MC DS-CDMA system in order to minimize the interference inflicted by the new user on already active users. Specifically, if there are subchannels that are not occupied by any other users, or that exhibit a sufficiently high SIR, the new user can use these passive subchannels or the subchannels exhibiting high SIR for transmitting data. However, if all the subchannels have been occupied and the SIR of each subchannel is insufficiently high, the new user can communicate by spreading its transmitted energy evenly across the subchannels. This transmission scheme imposes the minimum possible impact on the quality of service (QoS) of users already actively communicating. However, the simplest strategy for a new user to transmit is by randomly selecting one or several subchannels.

\section{Multirate and Variable Rate Services}

In FH/MC DS-CDMA systems multirate and variable-rate services can be implemented using a variety of approaches. Specifically, existing techniques, such as employing a variable spreading gain, multiple spreading codes, a variable constellation size, and variable-rate forward error correction (FEC) coding, can be invoked to provide multirate and variable rate services. Furthermore, since the proposed FH/MC DS- 


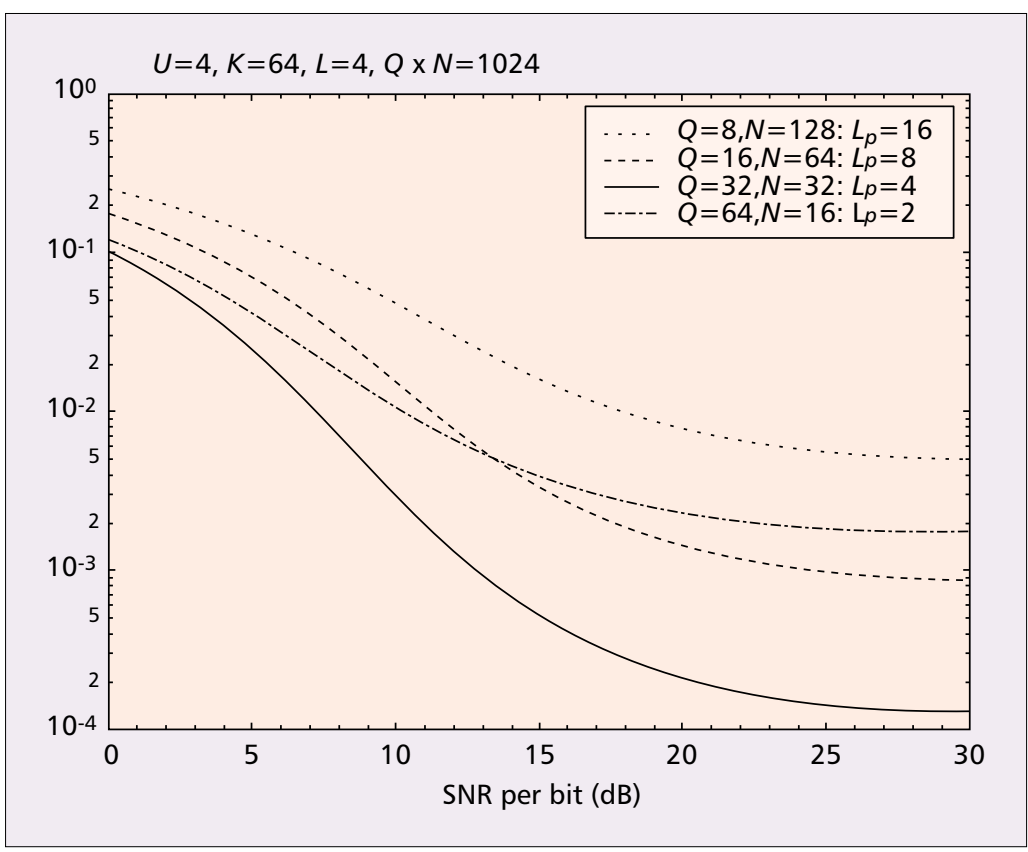

Figure 4. BER vs. SNR per bit performance for the constant-weight codebased SFH/MC DS-CDMA system varying the value of $\mathrm{N}$ and $\mathrm{Q}$ for a maximum diversity order of $\mathrm{L}=4$ and $\mathrm{L}_{\mathrm{p}}=2,4,8,16$. Given that the number of paths combined was $\mathrm{L}=4$, the system with $\mathrm{Q}=32, \mathrm{~N}=32, \mathrm{~L}=\mathrm{L}_{\mathrm{p}}=4$ achieved the best performance.

\section{INITIAL SYNCHRONIZATION}

In the FH/MC DS-CDMA system initial synchronization can be implemented by first accomplishing DS code acquisition. The fixed-weight code book index of the FH pattern used can be readily acquired, once DS code acquisition was achieved. During DS code acquisition the training signal supporting the initial synchronization, which is usually the carrier modulated signal without data modulation, can be transmitted using a group of subcarriers. These subcarrier signals can be combined at the receiver using, for example, equal gain combining (EGC) [8]. Hence, frequency diversity can be employed during the DS code acquisition stage of the FH/MC DS-CDMA system's operation, and consequently the initial synchronization performance can be significantly enhanced. Following the DS code acquisition phase, data transmission can be activated and the index of the FH pattern used can be signaled to the receiver using a given set of fixed subchannels. Alternatively, the index of the FH pattern can be acquired blindly from the received signal with the aid of a group of constant-weight codes having a given minimum distance [5].

\section{INTERFERENCE RESISTANCE}

The FH/MC DS-CDMA system concerned can mitigate the effects of intersymbol interference encountered during high-speed transmissions, and readily supports partial-band and multitone interference suppression. Moreover, multiuser interference can be suppressed by using multiuser detection techniques, potentially approaching single-user performance.

\section{AdVANCEd TeCHNOLOGIES}

The FH/MC DS-CDMA system can efficiently amalgamate the techniques of FH, OFDM, and DS-CDMA. Simultaneously, a variety of performance enhancement techniques, such as multiuser detection, turbo coding, adaptive antennas, space-time coding and transmitter diversity, and near-instantaneous adaptive modulation, might be introduced.

\section{FLEXIBILITY}

The future-generation broadband mobile wireless systems will aim to support a wide range of services and bit rates. The transmission rates may vary from voice and low-rate messages to very high-rate multimedia services requiring rates in excess of $100 \mathrm{Mb} / \mathrm{s}$ [9]. The communications environments vary in terms of their grade of mobility, the cellular infrastructure, the required symmetric and asymmetric transmission capacity, whether indoor, outdoor, urban, or rural area propagation scenarios are encountered, and so on. Hence air interfaces with the highest flexibility are required in order to maximize the area spectrum efficiency expressed in terms of bits per second per Hertz per square kilometer in a variety of communication environments. Future systems are also expected to support various types of services based on asynchronous transfer mode (ATM) transmission and the Internet Protocol (IP), which require varying QoS. As argued before, FH/MC DS-CDMA systems exhibit a high grade of com- 
patibility with existing CDMA systems. They benefit from the employment of $\mathrm{FH}, \mathrm{MC}$, and DS spreading. Furthermore, these systems also support the highest degree of freedom in the context of system design and parameter reconfiguration. In short, $\mathrm{FH} / \mathrm{MC}$ DS-CDMA systems constitute high-flexibility air interfaces.

Figure 4 shows that for a receiver combining a given number of resolvable paths, the average BER can be minimized by adjusting the number of subcarriers. In Fig. 4 FH/MC DS-CDMA systems having a constant system bandwidth associated with $N_{Q}=210$ were considered. However, the different scenarios used various combinations of the number of subcarriers $Q$ and bit duration to chip duration ratio of $N$. The number of active users was assumed to be $K=64$. It was also assumed that a receiver had a maximum diversity order of $L=4$, although a higher number of resolvable paths, $L_{p}$, was available at the receiver. From the results we infer that the configuration associated with $Q=32, N=32$, or $L=L_{p}=4$ achieves the best BER performance. Therefore, this example suggests that an FH/MC DS-CDMA system having a constant bandwidth can be optimized by adaptively reconfiguring the number of subcarriers as well as the corresponding DS spreading factors.

\section{Adaptive Rate Transmission}

\section{Why Adaptive Rate Transmission?}

There are a range of issues that motivate the application of adaptive rate transmission (ART) in the broadband wireless communication systems of the near future. The explosive growth of the Internet and the continued dramatic increase in demand for all types of wireless services are fueling the demand to increase user capacity, data rates, and the variety of services supported. Typical low-data-rate applications include audio conferencing, voice mail, messaging, email, and fax. Medium- to high-data-rate applications encompass file transfer, Internet access, highspeed packet- and circuit-based network access, as well as high-quality videoconferencing. Furthermore, broadband wireless systems are also expected to support real-time multimedia services, which provide concurrent video, audio, and data services for supporting advanced interactive applications. Hence, in the future generations of mobile wireless communication systems a wide range of information rates must be provided in order to support different services, which demand different data rates and different QoS. In short, an important motivation for using ART is to support a variety of services, which we refer to as service-motivated ART (S-ART). However, there is a range of other motivating factors, which are addressed below.

The performance of wireless systems is affected by a number of propagation phenomena:

- Path-loss variation vs. distance

- Random slow shadowing

- Random multipath fading

- Intersymbol interference (ISI), co-channel interference, as well as multiuser interference

- Background noise

For example, mobile radio links typically exhibit severe multipath fading, which leads to serious degradation in the link's SNR and consequently to a higher bit error rate (BER). In practice, fading compensation techniques such as using an increased link budget margin or interleaving combined with channel coding are typically required for improving the link's performance. However, today's cellular systems are designed for the worst-case channel conditions, typically achieving adequate voice quality over 90-95 percent of the coverage area for voice users, where the signal to interference plus noise ratio (SINR) is above the designed target [7]. Consequently, the systems designed for the worst-case channel conditions result in poor exploitation of the available channel capacity in case of good channel conditions. Adapting the transmitter's parameters in response to the timevarying channel conditions leads to better exploitation of the channel capacity available. This ultimately increases the achievable area spectral efficiency. Hence, the second reason for the application of ART is constituted by the time-varying nature of the channel, which we refer to as channel quality motivated ART (CART). All the above desirable system properties, including both S-ART and C-ART, can readily be supported with the aid of the proposed FH/MC DS-CDMA scheme. This will be outlined in more detail in the next section.

\section{What Is Adaptive Rate Transmission?}

Broadly speaking, ART in mobile wireless communications implies that the transmission rate at both the BSs and MSs can be adaptively adjusted according to instantaneous operational conditions, including the communication environment and service requirements. With the expected employment of SDR-based wireless systems, the concept of ART might also be extended to adaptively controlling the multiple access schemes - including FDMA, TDMA, narrowband CDMA, W-CDMA, and OFDM as well as the supporting network structures such as LANs and WANs. In this contribution, however, only C-ART and S-ART are considered in the context of the proposed FH/MC DS-CDMA scheme. Employing ART in response to different service requests indicates that the transmission rate of the BS and MS can be adapted according to the requirements of the services concerned, as well as to meet their different QoS targets. In contrast, employing ART in response to the time-varying channel quality implies that for a given service supported, the transmission rate of the BS and MS can be adaptively controlled in response to their near-instantaneous channel conditions. The main philosophy behind C-ART is the real-time balancing of the FH/MC DS-CDMA link budget through adaptive variation of the symbol rate, modulation constellation size and format, spreading factor, coding rate/scheme, and so on, or in fact any combination of these parameters, which is readily facilitated by the proposed FH/MC DS-CDMA scheme. Thus, by taking advantage of the time-varying nature of the wireless channel and interference conditions, C-ART schemes are capable of providing a significantly higher average spectral efficiency than their fixed-mode counterparts, provided that reliable
In future

generations of

mobile wireless

communication

systems, a wide

range of

information rates

must be

provided, in order

to support

different services.

In short, an

important

motivation for

using ART is to

support a variety

of services, which

we refer to as

service-motivated

ART (S-ART). 


A high number of
time slots might
be employed by a
user for achieving
a high diversity
gain, in order to
compensate for
the severe
channel fading
encountered with
the aid of using
an increased
spreading gain,
multiple transmis-
sion based
repetition coding
or a lower
channel
coding rate.

and low-latency channel state information can be acquired by the FH/MC DS-CDMA transmitter $[6,7]$. This takes place without wasting power, increasing the co-channel interference, or increasing the BER. We achieve these desirable properties by transmitting at high speeds under favorable interference/channel conditions and responding to degrading interference and/or channel conditions through a smooth reduction of the associated data throughput. Procedures that exploit the time-varying nature of the mobile channel are already in place for all the major cellular standards worldwide [7], including IS-95 CDMA, cdma2000 and UMTS W-CDMA, IS136 TDMA, the General Packet Radio Service of GSM and in the Enhanced Data Rates for Global Evolution (EDGE) schemes.

\section{ADAPTIVE RATE TRANSMISSION IN FH/MC DS-CDMA SYSTEMS}

The rudimentary portrayal of a range of existing and future ART schemes is given below. Note that a FH/MC DS-CDMA system may employ a combination of several ART schemes listed below, in order to achieve the desired data rate, BER or the highest possible area spectrum efficiency:

Multiple Spreading Codes: In terms of SART, higher-rate services can be supported by FH/MC DS-CDMA-based systems by assigning multiple spreading codes. In contrast, multiple codes cannot be employed in the context of CART in order to compensate for channel fading, path loss, and shadowing, since no diversity gain can be provided by signals transmitted by the same source over the same channel. However, if the co-channel interference is low - which usually implies in CDMA-based systems that the number of simultaneously transmitting users is low - multiple codes can be transmitted by an active user in order to increase the user's transmission rate.

Variable Spreading Factors: In the context of S-ART higher-rate services are supported by the FH/MC DS-CDMA system, when using lower spreading factors without increasing the bandwidth required. In terms of C-ART, when the SINR experienced is increased, reduced spreading factors are assigned to users for the sake of achieving higher data rates.

Variable-Rate FEC Schemes: The range of FEC coding schemes used in the FH/MC DSCDMA system might entail different classes of FEC codes, code structures, encoding/decoding schemes, coding rates, puncturing patterns, interleaving depths and patterns, and so on. In the context of S-ART, higher-rate services can be supported by coding schemes having a higher coding rate. In the context of C-ART, usually an appropriate coding scheme is selected by the FH/MC DS-CDMA system in order to maximize spectrum efficiency. The FEC schemes concerned may entail block or convolutional codes, block or convolutional constituent-code-based turbo codes, trellis codes, turbo trellis codes, and so on. The implementational complexity and error correction capability of these codes can be varied as a function of the coding rate, code constraint length, number of turbo decoding itera- tions, puncturing pattern, and so on. A rule of thumb is that the coding rate is increased toward unity, as the channel quality improves, in order to increase the system's effective throughput.

Variable Constellation Size: In S-ART schemes higher-rate services can be supported by the FH/MC DS-CDMA system upon transmitting multibit symbols associated with higher constellation sizes. For example, an adaptive FH/MC DS-CDMA modem may employ binary phase shift keying (BPSK), quaternary PSK (QPSK), 16-quadrature amplitude modulated (QAM), and 64-QAM constellations, which corresponds to $1,2,4$, and $6 \mathrm{~b} /$ symbol. The highest data rate provided by the 64-QAM constellation is a factor of six higher than that provided employing the BPSK constellation. In C-ART scenarios, when the SINR increases, a higher number of bits per symbol associated with a higher order constellation is transmitted for increasing the system's throughput.

Multiple Time Slots: In an S-ART scenario higher rate services can also be supported by the FH/MC DS-CDMA system upon assigning a corresponding number of time slots. A multipletime-slot-based adaptive rate scheme has been used in GPRS-136 (1-3 time slots/20 ms) and in Enhanced GPRS (EGPRS) (1-8 time slots/4.615ms GSM frame) in order to achieve increased data rates. In the context of C-ART, multiple time slots associated with interleaving or FH can be implemented for achieving time diversity gain. Hence, C-ART can be implemented in the context of FH/MC DS-CDMA as follows. A high number of time slots might be employed by a user to achieve a high diversity gain in order to compensate for the severe channel fading encountered with the aid of using an increased spreading gain, multiple transmission based repetition coding, or a lower channel coding rate, for example. Then, under the constraint of a given total number of time slots employed by a user the effective throughput of the user must be reduced. In contrast, assigning a low number of time slots over benign nonfading channels allows us to achieve high effective throughput, since spreading gain can be reduced, or the channel coding rate can be increased, for example.

Multiple Bands: In the context of S-ART higher-rate services can also be supported by the FH/MC DS-CDMA system by assigning a higher number of frequency bands. In the context of CART associated with multiple bands, FH associated with time-variant redundancy and/or variable rate FEC coding schemes, or frequency diversity techniques might be invoked, in order to increase the spectrum efficiency of the system. For example, in C-ART schemes associated with doubleband assisted frequency diversity, if the channel quality is low, the same signal can be transmitted in two frequency bands for the sake of maintaining a diversity order of two. However, if the channel quality is sufficiently high, two independent streams of information can be transmitted in these bands and consequently the throughput of the FH/MC DS-CDMA system can be doubled.

Multiple Transmit Antennas: Employing multiple transmit antennas based on space-time coding (STC) and space-time spreading (STS) is a novel method of communicating over wireless 
channels, which was also optionally adapted for employment in the $3 \mathrm{G}$ mobile wireless systems [10]. ART in the FH/MC DS-CDMA system can also be implemented using multiple transmit antennas associated with different STC or STS schemes. In S-ART schemes higher-rate services can be supported by a higher number of transmit antennas associated with appropriate STC or STS schemes. In terms of C-ART schemes, multiple transmit antennas in the FH/MC DSCDMA systems can be invoked to achieve high diversity gain, but reduced throughput. Therefore, when the channel quality expressed in terms of the SINR is sufficiently high, the diversity gain can be decreased. Consequently, two or more symbols can be transmitted in each signaling interval, and each stream is transmitted by only a fraction of the transmit antennas associated with the appropriate STC or STS schemes. Hence the throughput is increased. However, when the channel quality is poor, an increased number of transmit antennas or even all the transmit antennas can be activated for transmitting a single stream of data, hence achieving the highest possible transmit diversity gain of the system while decreasing the throughput.

Above we have summarized the philosophy of a number of ART schemes, which can be employed in adaptive FH/MC DS-CDMA-based wireless communication systems. An S-ART scheme requires a certain level of transmitted power in order to achieve the required QoS. Specifically, relatively high transmitted power is necessitated for supporting high-data-rate services, and a relatively low transmitted power is required for offering low-data-rate services. Hence, a side-effect of an S-ART scheme supporting high-data-rate services is the concomitant reduction of the number of users supported due to increased interference or/and increased bandwidth. In contrast, a cardinal requirement of a C-ART scheme is accurate channel quality estimation or prediction at the receiver as well as provision of reliable side-information feedback between the channel quality estimator or predictor of the receiver and the remote transmitter [6,7], where the modulation/coding mode requested by the receiver is activated. The parameters capable of reflecting the channel quality may include BER, SINR, transmission frame error rate, received power, path loss, automatic repeat request (ARQ) status, and so on. A C-ART scheme typically operates under the constraint of constant transmit power and constant bandwidth. Hence, without wasting power and bandwidth, or increasing the co-channel interference compromising BER performance, C-ART schemes are capable of providing significantly increased average spectral efficiency by taking advantage of the time-varying nature of the wireless channel when compared to fixedmode transmissions. The cost for successfully using C-ART is the associated increase of complexity due to channel state estimation or prediction as well as due to the channel state information feedback to be conveyed from the receiver to the transmitter. Furthermore, a fraction of the channel capacity has to be used for transmitting the feedback information. However, the capacity loss due to channel state informa- tion feedback is modest, provided that the fading is not too rapid and the transmission burst duration is not excessively short [6].

\section{SOFTWARE-DEFINED-RADIO-ASSISTED FH/MC DS-CDMA}

According to our arguments in the previous sections, broadband FH/MC DS-CDMA constitutes a versatile broadband air-interface, which provides a wide range of flexible parameters that may be reconfigured in response to the prevalent service requirements and/or communication environments. Reconfiguration of the broadband FH/MC DS-CDMA systems can be conveniently implemented using SDRs [11]. Furthermore, different legacy systems will continue to coexist, unless the ITU - by some miracle - succeeds in harmonizing all the forthcoming standards under a joint framework, while at the same time ensuring backward compatibility with the existing standards. The range of existing wireless communication systems is shown in Fig. 5. Therefore, in the absence of the "ultimate" standard, the only solution is employing multiband, multimode, multistandard transceivers based on the concept of SDR. Although broadband FH/MC DS-CDMA systems in the short term have the potential to maintain compatibility with the existing $2 \mathrm{G}$ and $3 \mathrm{G}$ CDMA systems, in the long term it is inevitably required to be compatible with the other standards. Therefore, the SDRs used for implementing broadband FH/MC DS-CDMA systems are not only instrumental in terms of supporting parameter reconfiguration, but also capable of supporting interworking of CDMAbased systems with non-CDMA-based standards.

In SDRs the digitization of the received signal is performed at some stage downstream from the antenna, typically after wideband filtering, low-noise amplification, and down-conversion to a lower frequency. The reverse processes are invoked by the transmitter. In contrast to most wireless communication systems that employ digital signal processing (DSP) only at baseband, SDRs are expected to implement DSP functions at an intermediate frequency (IF) band. An SDR defines all aspects of the air interface, including $\mathrm{RF}$ channel access and waveform synthesis, in software. In SDRs wideband analog-to-digital and digital-to-analog converters (ADCs and DACs) transform each RF service band from digital and analog forms at IF. The wideband digitized receiver stream of bandwidth $W_{s}$ accommodates all subscriber channels, each of which has a bandwidth of $W_{c}\left(W_{c}<<W_{s}\right)$. Thanks to using programmable DSP chips at both the IF and baseband, SDRs are sufficient to efficiently support multiband and multistandard communications. An SDR employs one or more reconfigurable processors embedded in a real-time multiprocessing fabric, permitting flexible reprogramming and reconfiguration using software downloaded, for example, with the aid of a signaling channel from the BS. Hence, SDRs offer an elegant solution to accommodating various modulation formats, coding, and radio access schemes. They also have the potential of reducing the cost of introducing new technology superceding legacy systems, and are
Re-configuration

of the broadband

FH/MC DS-CDMA

systems can be

conveniently

implemented

using Software

Defined Radios.

Furthermore, different legacy

systems will

continue to

coexist, unless

the ITU succeeds

in harmonizing all

the forthcoming

standards under a

joint framework. 
FH/MC DS-CDMA

systems will be

designed in order

to provide the

maximum grade

of compatibility

with the existing

CDMA based

systems, such as

IS-95 and

W-CDMA

based systems.

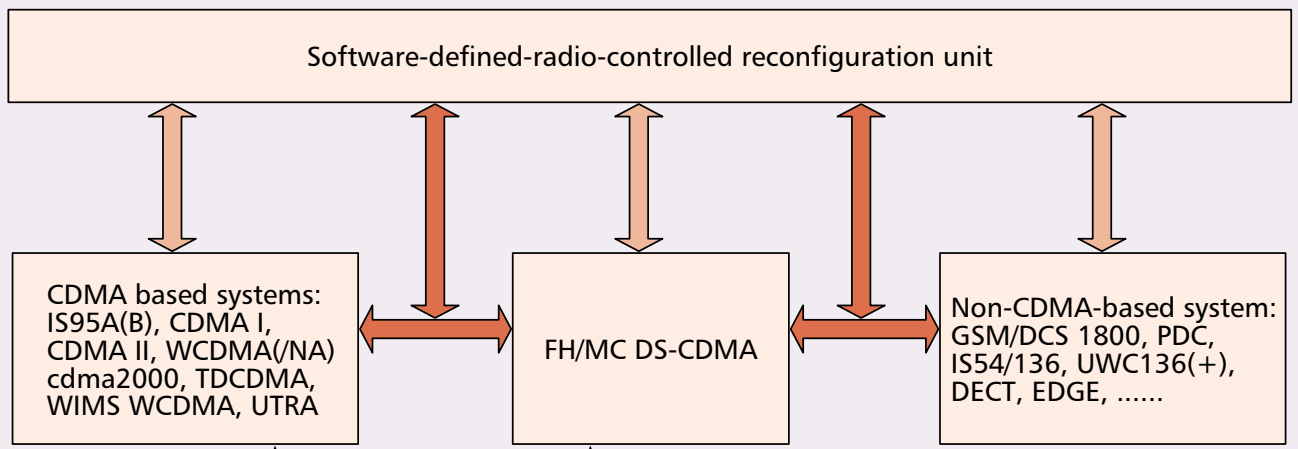

Compatibility

IS-95: Digital cellular standards in the United States IS-95B: Enhanced version of IS-95

CDMA I: Multiband synchronous DS-CDMA

CDMA II: Asynchronous DS-CDMA

W-CDMA: Wideband CDMA

W-CDMA/NA: North American W-CDMA

cdma2000: Multicarrier CDMA system based on IS-95

TDCDMA: Time-division synchronous CDMA

WIMS W-CDMA: Wireless multimedia and messaging services W-CDMA

UTRA: UMTS Terrestrial Radio Access

GSM: Global System for Mobile Communications

DCS1800: GSM system in the $1800 \mathrm{MHz}$ band

PDC: Japanese personal digital cellular system

IS-54: American digital advanced mobile phone system (DAMPS)

IS-136: North American TDMA system

UWC136: Universal wireless communications based on IS136

UWC136+: Enhanced version of UWC136

DECT: Digital Enhanced Cordless

Telecommunications

EDGE: Enhanced Data Rates for Global Evolution

Figure 5. Software-defined radio assisted FH/MC DS-CDMA and its reconfiguration modes.

amenable to future software upgrades, potentially supporting sophisticated future signal processing functions such as array processing, multiuser detection, and as yet unknown coding techniques.

FH/MC DS-CDMA systems will be designed in order to provide the maximum grade of compatibility with the existing CDMA-based systems, such as IS-95 and W-CDMA-based systems. For example, the frequency bands of the IS-95 CDMA system in North America are 824-849 $\mathrm{MHz}$ (uplink) and 869-894 MHz (downlink), respectively. The corresponding frequency bands for the UMTS-FDD W-CDMA system are 1850$1910 \mathrm{MHz}$ (uplink) and 1930-1990 MHz (downlink) in North America, while 1920-1980 MHz (uplink) and 2110-2170 MHz (downlink) in Europe. In order to ensure compatibility with these systems, the proposed FH/MC DS-CDMA system's spectrum can be assigned according to Fig. 6. Specifically, in the frequency band of IS95, 39 orthogonal subcarriers are assigned, each having a bandwidth of $1.25 \mathrm{MHz}$, while in the frequency band of UMTS-FDD W-CDMA, 23 orthogonal subcarriers are allocated, each with a bandwidth of $5 \mathrm{MHz}$. The MC modulation used in the FH/MC DS-CDMA system obeying the above spectrum allocation can readily be implemented using two IFFT subsystems at the transmitter and two FFT subsystems at the receiver, where a pair of IFFT-FFT subsystems carries out modulation/demodulation in the IS-95 band, while another pair of IFFT-FFT subsystems transmits and receives in the UMTS-FDD band. If the chip rate for the $1.25 \mathrm{MHz}$ bandwidth subcarriers is $1.2288 \mathrm{M}$ chips/s and for the $5 \mathrm{MHz}$ bandwidth subcarriers 3.84 Mchips/s, the FH/MC DS-CDMA system will be compatible with both the IS-95 and UMTS-FDD W-CDMA systems.

However, the terminals of future broadband wireless systems are expected not only to support multimode and multistandard communications, but also to possess the highest possible grade of flexibility while achieving high spectrum efficiency. Hence, these systems are expected to be capable of software reconfiguration both between different standards as well as within a specific standard. In contrast to reconfiguration between different standards invoked, mainly for the sake of compatibility, the objective of reconfiguration within a specific standard is to support a variety of services at the highest spectrum efficiency. The SDR-assisted broadband FH/MC DS-CDMA system is operated under the control of the software reconfiguration unit shown in Fig. 5. The set of reconfigured parameters of the broadband FH/MC DS-CDMA system may include:

- Services: Data rate, QoS, real-time or nonreal-time transmission, encryption/decryption schemes and parameters

- Error control: CRC, FEC codes, coding/decoding schemes, coding rate, number of turbo decoding steps, interleaving depth and pattern

- Modulation : Modulation schemes, signal constellation, partial response filtering

- PN sequence : Spreading sequences (codes), chip rate, chip waveform, spreading factor, PN acquisition and tracking schemes

- Frequency hopping: FH schemes (slow, fast, random, uniform, and adaptive), FH patterns, weight of constant-weight codes

- Detection: Detection schemes (coherent or noncoherent, etc.) and algorithms (maximum likelihood sequence detection, MLSD, or minimum mean square estimation, MMSE, etc.), parameters associated with space/time as well as frequency diversity, beamforming, diversity combining schemes, equalization schemes as well as the related parameters (such as the number of turbo 


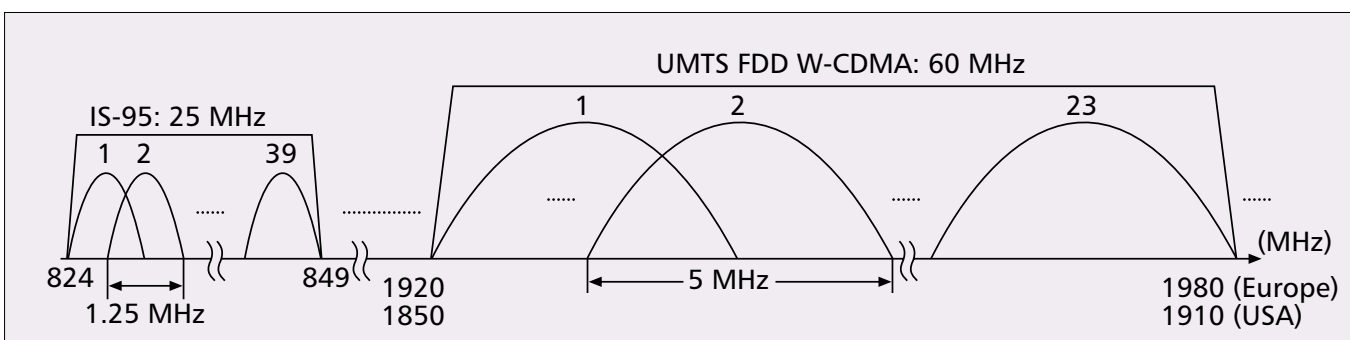

(a) Uplink

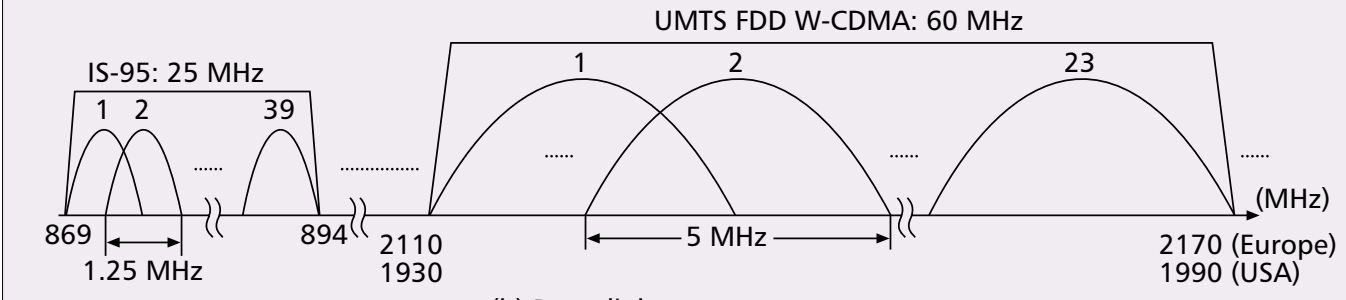

(b) Downlink

Figure 6. Exhibition of spectrum compatibility of the broadband FH/MC DS-CDMA system with IS-95 and UMTS-FDD wideband CDMA systems.

equalization iterations, etc.) and channel quality estimation algorithms, parameters

- Others: Subchannel bandwidth, power control parameters

In the context of different standards - in addition to the parameters listed above - the transceiver parameters that must be reconfigurable have to include the clock rate, radio frequency (RF) bands, and air interface modes.

\section{FINAL REMARKS}

We have presented a flexible broadband mobile wireless communication system based on FH/MC DS-CDMA and reviewed the existing as well as a range of forthcoming techniques, which might be required to develop broadband mobile wireless systems exhibiting high flexibility and high efficiency. We argued that this broadband FH/MC DS-CDMA system exhibits a high grade of compatibility with the existing CDMA-based systems, since it is capable of incorporating a wide range of techniques developed for the $2 \mathrm{G}$ and $3 \mathrm{G}$ mobile systems. At the time of writing research is well underway toward the SDR-based implementation of a range of existing systems. It is expected that these efforts will soon encompass a generic scheme not too different from the FH/MC DSCDMA scheme advocated in this contribution.

\section{REFERENCES}

[1] M. Zeng, A. Annamalai, and V. K. Bhargava, "Recent Advances in Cellular Wireless Communications," IEEE Commun. Mag., Sept. 1999, pp. 128-38.

[2] P. Chaudhury, W. Mohr, and S. Onoe, "The 3GPP proposal for IMT-2000," IEEE Commun. Mag., Dec. 1999, pp. 72-81.

[3] R. Steele and L. Hanzo, Eds., Mobile Radio Communications, IEEE Press-John Wiley, 2nd ed., 1999.

[4] L.-L. Yang and L. Hanzo, "Slow Frequency-hopping Multicarrier DS-CDMA over Nakagami Multipath Fading Channels," IEEE JSAC, vol. 19, no. 7, July 2001, pp. 1211-21.

[5] L.-L. Yang and L. Hanzo, "Blind Joint Soft-detection Assisted Slow Frequency-Hopping Multicarrier DS-CDMA," IEEE Trans. Commun., vol. 48, Sept. 2000, pp. 1520-29.
[6] M. S. Alouini and A. J. Goldsmith, "Capacity of Rayleigh Fading Channels under Different Adaptive Transmission and Diversity-combining Techniques," IEEE Trans. Vehic. Tech., vol. 48, July 1999, pp. 1165-81.

[7] S. Nanda, K. Balachandran, and S. Kumar, "Adaptation Techniques in Wireless Packet Data Services," IEEE Commun. Mag., Jan. 2000, pp. 54-64.

[8] L.-L. Yang and L. Hanzo, "Parallel Code-acquisition for Multicarrier DS-CDMA Systems Communicating over Multipath Nakagami Fading Channels,"' Proc. IEEE GLOBECOM 2000, San Francisco, CA, Nov. 27- Dec. 1, 2000.

[9] M. Progler, C. Evci, and M. Umehira, "Air Interface Access Schemes for Broadband Mobile Systems," IEEE Commun. Mag., Sept. 1999, pp. 106-15.

[10] B. Hochwald, T. L. Marzetta, and C. B. Papadias, "A Transmitter Diversity Scheme for Wideband CDMA Systems based on Space-time Spreading," IEEE JSAC, vol. 19, Jan. 2001, pp. 48-60.

[11] J. Mitola. III, "Technical Challenges in the Globalization of Software Radio," IEEE Commun. Mag., Feb. 1999, pp. 84-89.

\section{ADDITIONAL READING}

[1] Special issue on Software Radios, IEEE JSAC, vol. 17, no. 4, Apr. 1999, pp. 509-747.

[2] L. Hanzo, C. H. Wong, and M. S. Yee, Adaptive Wireless Transceivers, John Wiley-IEEE Press, 2002.

\section{BIOGRAPHIES}

LIE-LIANG YANG received his B.Eng. degree in communication engineering from Shanghai TieDao University, Shanghai, China, and his M.S. and Ph.D. degrees in communications and electronics from Northern Jiaotang University, Beijing, China, in 1988, 1991, and 1997, respectively. Since December 1997 he has been with the Communication Group. Department of Electronics and Computer Science, University of Southampton, United Kingdom. He has published over 60 papers in journals and conference proceedings and was awarded the Royal Society Sino-British Fellowship in 1997.

LAJOS HANZO (http://www-mobile.ecs.soton.ac.uk) graduated in electronics in 1976 and in 1983 received his doctorate. During his 25-year career in telecommunications he has held various research and academic posts in Hungary, Germany, and the United Kingdom. Since 1986 he has been with the Department of Electronics and Computer Science, University of Southampton, and has been a consultant to Multiple Access Communications Ltd., United Kingdom. Currently he holds the chair of telecommunications at the University of Southampton.
Research is well

under way

toward the

SDR-based

implementation

of a range of

existing systems.

It is expected that

these efforts will

soon encompass

a generic scheme not too different

from the FH/MC

DS-CDMA scheme advocated in this

contribution. 